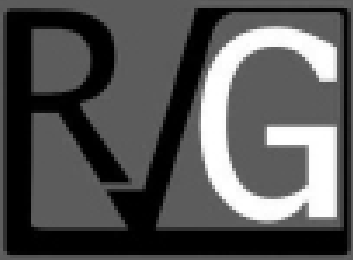

Año 24 No. 88

Octubre - Diciembre 2019

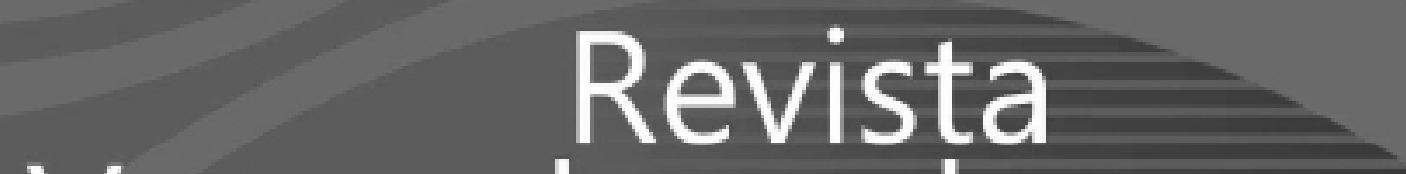

Venezolana de

verencla

UNIVERSIDAD DEL ZULIA (LUZ)

Facultad de Ciencias Económicas y Sociales

Centro de Estudios de la Empresa 


\title{
Liderazgo y riesgo psicosocial en instituciones de educación superior en México
}

\author{
Saldaña Orozco, Claudia ${ }^{1}$ \\ Bustos Saldaña, Rafael ${ }^{2}$ \\ Barajas Martínez, Alfonso ${ }^{3}$ \\ Ibarra Rentería, Guadalupe Montserrat ${ }^{4}$
}

\begin{abstract}
Resumen
El liderazgo es visto como una influencia interpersonal ejercida ante determinadas situaciones, esto con el fin de alcanzar uno o diversos objetivos. Siendo visto como factor fundamental para el éxito de una organización. Sin embargo, cuando este no está presente en el ambiente laboral surgen los Factores de Riesgo Psicosocial. Además, cuando existe la ausencia de liderazgo las exigencias comienzan a sobrepasar las capacidades del trabajador produciendo agotamiento, depresión o estrés laboral. Con la presente investigación se busca determinar la relación entre el estrés y los factores de riesgo psicosocial y de estos con los estilos de liderazgo. Se aplicó un cuestionario de liderazgo, la Norma Mexicana 035 y el cuestionario de estrés a personal operativos y administrativos del Centro Universitario del Sur. Al realizar una correlación entre las variables se muestra un aumento de estrés y factores de riesgo psicosocial en
\end{abstract}

Recibido: 15-05-19

Aceptado: 12-09-19

1 Docente investigadora en el Centro Universitario del Sur México, miembro del Sistema Nacional de Investigación, Autora de libros, autora de artículos en revistas nacionales e internacionales, ponente, arbitro de revistas internacionales, Estratega, consultora de organizaciones públicas y privadas.claudias@cusur.udg.mx,https://orcid.org/0000-0003-3268-7209

2 Maestro en Ciencias Médicas, profesor investigador Titular B, miembro del Cuerpo Académico de Salud Pública del Centro Universitario del Sur. rafaelb@cusur.udg.mx; https://orcid.org/00000001-8357-3610

3 Profesor docente Titular C, miembro del Cuerpo Académico de Salud Pública del Centro Universitario del Sur.barajas@cusur.udg.mx,https://orcid.org/0000-0002-6694-2565

4 Estudiante de la Lic. Psicología, becaria académica en investigación del Centro Universitario del Sur. gmontse.ibre97@gmail.comhttps://orcid.org/0000-0001-9603-6179 
presencia de Laissez Faire y disminuyendo en presencia de liderazgo transformacional o transaccional. Concluyendo que el estilo de liderazgo juega un papel importante en la salud de los trabajadores.

Palabras clave: estrés laboral; factores de riesgo psicosociales; liderazgo.

\title{
Leadership and Psychosocial Risk in Higher Education Institutions in Mexico
}

\begin{abstract}
Leadership is seen as an interpersonal influence exerted in certain situations, this in order to achieve one or several objectives. Being seen as a fundamental factor for the success of an organization. However, when it is not present in the work environment, Psychosocial Risk Factors arise. In addition, when there is the absence of leadership, the demands begin to exceed the worker's abilities, causing exhaustion, depression or work stress. This research seeks to determine the relationship between stress and psychosocial risk factors and these with leadership styles. A leadership questionnaire, Mexican Standard 035 and the stress questionnaire were applied to operational and administrative personnel of the University Center of the South. When correlating the variables, an increase in stress and psychosocial risk factors is shown in the presence of Laissez Faire and decreasing in the presence of transformational or transactional leadership. Concluding that leadership style plays an important role in workers' health
\end{abstract}

Keywords: work stress; psychosocial risk factors; leadership.

\section{Introducción.}

La Organización Mundial de la Salud expone que el personal administrativo de una institución de educación superior debe ir a la par del desarrollo dinámico y productivo del mundo de hoy, considerado como el talento humano que contribuye al desenvolvimiento de los procesos administrativos porque es la imagen institucional, justamente por estar en contacto, tanto con los usuarios internos y externos, como por ende las exigencias y la constante presión son parte de la cotidianidad del trabajo administrativo; a veces esta presión se hace excesiva o difícil de controlar (OMS, 2004).

Esto se expone de una mejor manera en el Reglamento Federal De Seguridad y Salud en el Trabajo el cual refiere la existencia de riesgos para la salud en el lugar de trabajo, incluidos el calor, el ruido, el polvo, los productos químicos peligrosos, las máquinas inseguras y el estrés psicosocial provoca enfermedades ocupacionales y pueden agravar otros problemas de salud 
(Diario Oficial de la Federación, 2014). Cabe destacar que en el presente año la Organización para la Cooperación y el Desarrollo Económicos (OCDE) menciona que el 43 por ciento de los mexicanos padece estrés laboral, siendo el país con mayores índices a nivel global; además, los mexicanos son los que más número de horas trabajan a la semana. Esto combinado con la falta de liderazgo hace más propensos a los trabajadores a Riesgos Psicosociales (OMS, 2017).

Debido a estas cifras el objetivo de la investigación consiste en determinar la relación entre los Factores de Riesgo Psicosocial (FRP) y el Estrés (EST) en el personal operativo y administrativo del Centro Universitario del Sur, y de qué manera estos aspectos se relacionan con el estilo de liderazgo ejercido. Para lograr el objetivo se analizarán en un inicio los 3 factores a estudiar (LIDER, FRP y EST) y su presencia en el grupo de estudio, posteriormente se pasará a analizar si existe una relación entre ellos y de qué manera pueden llegar a afectar a los trabajadores si se encuentran en manera conjunta o aislada.

Se seleccionó el Centro Universitario del Sur ubicada en Zapotlán el Grande, Jalisco. La muestra se conformó por 114 personas del personal operativo y administrativo del Centro Universitario donde se encuentran 47 hombres y 67 mujeres, los cuales se encontraban entre los 21 y los 67 años de edad. La muestra fue elegida a conveniencia. Para el estudio se llevó a cabo una investigación teórica en una primera instancia, posteriormente fue seleccionada una metodología cuantitativa, no experimental con diseño correlacional utilizando el programa SPSS versión 24 para dichas correlaciones. Se contó con la aplicación de tres instrumentos.

El primero, fue el Multifactor Leadership Questionnaire, (Bass y Avolio, 2014), el segundo instrumento es el cuestionario contenido en la Norma mexicana de prevención de riesgos psicosociales (NOM 035, 2018), y el tercero consistió en la aplicación del cuestionario de Estrés, propuesto por la Organización Internacional del Trabajo y por la Organización Mundial para la Salud (OIT/OMS, 2007).

Posteriormente, con la información recolectada y con ayuda del programa SPSS versión 24 se llevará a cabo una correlación bi variada, con el fin de comparar dichos resultados y ser contrastados con el objetivo con el fin de validar o refutar la relación existente entre estos tres factores. A partir de lo anterior se presentarán los hallazgos y las conclusiones de la investigación, donde también se incluirán las sugerencias de métodos de intervención para los trabajadores del Centro Universitario. Debido a las pocas investigaciones realizadas a esta población en particular, supone un aporte valioso tanto en la parte teórica como en los resultados.

\section{Liderazgo, Factores de Riesgo Psicosocial y Estrés: consideraciones teóricas.}

Para entender el concepto de liderazgo y la manera en la que se presenta se consultó el Modelo de Liderazgo utilizado por Bass y Avolio (1995), el cual explica que un líder puede alternar entre diferentes tipos de liderazgos según la demanda de las situaciones específicas del entorno y las expectativas de los miembros de la organización.

Estos tipos de liderazgo se dividen 
en tres, el primero es el liderazgo transaccional el cual es definido como un intercambio entre el líder y sus seguidores, tomando una relación costobeneficio (Bass, 1999), el liderazgo transformacional son conocidos como transformadores ya que a través de la influencia ejercida sobre los miembros del grupo, estimula cambios que llevan del interés individual al colectivo (Bass, 1985), y por último el LaissezFaire también conocido como "dejar de hacer", ofrece pocos términos de dirección y soporte, evita la toma de decisiones, es desordenado dejando a sus subordinados hacer lo que les parece, presentando indiferencia ante las acciones de los demás (Bass y Avolio, 2004).

El Multifactor Leadership Questionnaire (MLQ) creado para la medición de liderazgo y tomando como principio la teoría de Bass y Avolio, utiliza como base el modelo Full Range Leadership (FRL) esquematizado por Mendoza Martínez (2005), el cual maneja los siguientes atributos (Cuadro 1)

\section{Cuadro 1 \\ Tipos de liderazgo y características}

\begin{tabular}{ccl}
\hline Tipo de liderazgo & & \multicolumn{1}{c}{ Características. } \\
\hline \multirow{2}{*}{ Liderazgo transformacional } & 1. & Atributos. \\
& 2. & Conducta. \\
& 3. & Estimulación intelectual. \\
& 4. & Consideración individualizada. \\
& 5. & Tolerancia sociológica \\
\hline \multirow{2}{*}{ Liderazgo transaccional } & 6. & Premio contingente. \\
& 7. & Excepción activa. \\
& 8. & Excepción pasiva. \\
\hline No liderazgo & 9. & Laissez- Faire \\
\hline
\end{tabular}

Fuente: Elaboración propia

Otro aspecto al que se encuentran expuestos el personal operativo $y$ administrativo de las instituciones, son los Factores de Riesgo Psicosocial (FRPS), los cuales son definidos por la Organización Internacional del Trabajo (1998) como las interacciones entre el trabajo, su medio ambiente, así como la satisfacción en el trabajo y las condiciones de organización, teniendo en cuenta las capacidades del trabajador, sus necesidades, su cultura y su situación personal fuera del trabajo que pueden influir en el estado de salud y bienestar de las personas, hasta determinar su nivel de rendimiento, además de su satisfacción en el trabajo.

Tal dimensión comprende los aspectos psicológicos y sociales que subyacen en la actividad laboral que desempeña un trabajador y afectan la calidad de vida de los mismos. Estos factores psicosociales pueden favorecer o perjudicar la actividad laboral y la calidad de vida laboral de las personas. En el primer caso, fomentan el desarrollo 
personal de los individuos, mientras que cuando son desfavorables perjudican su salud y su bienestar. En este caso se habla de que el riesgo psicosocial es fuente de estrés laboral, o estresor y que tiene el potencial de causar daño psicológico, físico, o social a los individuos (Díaz eta al., 2016).

Finalmente, el estrés laboral se ve presente cada vez con mayor frecuencia entre los trabajadores ya que entre el $5 \%$ y el $10 \%$ de los trabajadores en los países desarrollados sufren de estrés laboral, mientras que en los industrializados sería el $20 \%$ y el $50 \%$ los trabajadores afectados (OMS, 2017), esto debido a las condiciones poco saludables o riesgosas a las que se enfrentan en el ambiente laboral. Al presentarse una situación de estrés el individuo puede tornarse irritado, desconcentrado, cansado e intranquilo llevando al trabajador a un bajo rendimiento o al ausentismo laboral (Viera, 2014). Además, según el Informe de Observación Europeo a través de encuestas a 40 millones de personas, se concluyó que las personas que sufrían mayores índices de estrés están presentes en la mano de obra (22\%), sector de educación y salud $(12.7 \%)$ y administrativos (11.1\%) (Hassard J, Teoh K, et al, 2015), debido a estos datos la población elegida se centrará en trabajadores del sector educativo.

\section{Liderazgo y riesgo psicosocial en instituciones de educación superior en México: Relaciones}

La presente investigación se llevó a cabo en el Centro Universitario del Sur, ubicada en Zapotlán el Grande, Jalisco. La muestra se conformó por 114 personas pertenecientes al personal operativo y administrativo, siendo elegida bajo conveniencia. Fueron registrados 47 hombres y 67 mujeres, los cuales se encontraban entre los 21 y los 67 años de edad.

La investigación se centró en el personal administrativo (70.18\%) quien de acuerdo con de acuerdo a BatemanSnell (2001), sus funciones principales son: organización, planeación, dirección y control; y personal operativo $(42.98 \%)$ el cual comprende al personal cuyo trabajo está relacionado directamente con los procesos de abastecimiento, producción y distribución, excepto aquél cuyas labores son de dirección o supervisión técnica y administrativa. Según Villegas (1996) representa el activo más valioso para las empresas, puesto que es a través de su actividad laboral diaria como se pueden lograr los objetivos organizacionales.

Para el estudio fue seleccionada una metodología cuantitativa, no experimental con diseño correlacional. Se contó con la aplicación de tres instrumentos. El primero fue el Cuestionario de Liderazgo (MQL) (Bass y Avolio, 2004), con el cual se midieron tres tipos de liderazgo los cueles son, Liderazgo Transformacional, Liderazgo Transaccional y el Laissez Faire. Con el instrumento se pretendía evaluar la tolerancia psicológica como un aspecto que conforma el Liderazgo Transformacional, así como las variables efectividad, satisfacción y esfuerzo extra. El cuestionario MLQ ha demostrado una confiabilidad entre 0.85 y 0.96 en sus diferentes dimensiones y varianzas explicadas superiores a 58.9 (Edinson et al, 2019).

El segundo instrumento consistió en el cuestionario contenido en la Norma mexicana de prevención de riesgos psicosociales (NOM 35, 2018), con 
la que se evaluaron los dominios de Ambiente de Trabajo, Factores Propios de la Actividad, Organización del Tiempo de Trabajo y el Liderazgo y las Relaciones de Trabajo. Se determinó el Alfa de Cronbach total en este estudio $\alpha=.885$.

Finalmente, el cuestionario de EST propuesto por la Organización Internacional del Trabajo y por la Organización Mundial para la Salud (OIT/OMS, 2016), que mide dos factores de EST: condiciones organizacionales y procesos administrativos. Los autores del cuestionario reportaron el $64 \%$ de validez relevante, con $43.55 \%$ de varianza explicada y 0.92 de confiabilidad por Alpha de Cronbach. En este estudió se calculó un alfa de Cronbach total $\alpha=$ 0.95 .

Para facilitar el orden de los descubrimientos se plantearon 4 hipótesis las cuales se pretenden responder con los resultados obtenidos. Las hipótesis son las siguientes:

Hipótesis 1: en el Centro Universitario del Sur (CUSur) existe la presencia de las tres variables que se están evaluando en la investigación (liderazgo, Factores de Riesgo Psicosocial y Estrés) y hay diferencias entre personal operativo y administrativo.

Hipótesis 2: existe una relación entre el estilo de liderazgo y la aparición de FRP y EST (esto será comprobado con ayuda de correlaciones bi variadas llevadas a cabo con el programa SPSS versión 24).

Hipótesis 3: existe una relación entre la presencia de liderazgo Laissez Faire (LLF) y la aparición del estrés (EST) así como de los Factores de Riesgo (FRP) en los trabajadores del CUSur.

Hipótesis 4: cuando existe la presencia de liderazgo Transformacional (LTF) o Transaccional (LTS), el EST y los FRP se van a encontrar presentes en menor medida.

Luego de la aplicación de los instrumentos, se continuó con el análisis de los resultados obtenidos en la población para comprobar la hipótesis 1 . Los resultados se muestran en la tabla 1.

\section{Tabla 1. \\ Comparación de personal operativo y administrativo en la aplica- ción de instrumentos

\begin{tabular}{lccc}
\hline Instrumento & Administrativos & Operativos & Significancia \\
\hline Estrés & 46.67 & 45.18 & NS \\
\hline MLQ & 157.13 & 129.88 & 0.0138 \\
\hline NOM035 & 62.038 & 74.088 & 0.01232 \\
\hline
\end{tabular}

Fuente: elaboración propia

Como se puede observar en la tabla 1 , los resultados obtenidos muestran que los niveles de estrés mantuvieron en un nivel bajo, por lo cual no fue significativo. Sin embargo, tanto el cuestionario MLQ y la NOM 035 mostraron datos significativos, los cuales indican el nivel de liderazgo y la presencia de FRP en el ambiente de trabajo presentándose en mayor medida 
en el personal administrativo que en el personal operativo.

Posteriormente y con ayuda de programa SPSS versión 24 se realizó un análisis de los resultados obtenidos por los instrumentos, esta vez se utilizó la correlación bi -variada, con el fin de conocer si existía una relación entre las tres variables y así respaldar las tres hipótesis restantes. Los resultados se muestran en la tabla 2 a continuación.

Tabla 2.

Relación entre variables

\begin{tabular}{|c|c|c|c|c|c|c|c|c|c|}
\hline & EOR & EAA & LTF & LTS & LLF & FCAT & FCT & FFC & FOT \\
\hline EAA & $.808^{* *}$ & & & & & & & & \\
\hline LTF & $-.430^{* *}$ & $-.286^{\star *}$ & & & & & & & \\
\hline LTS & $-.339^{* *}$ & $-.260^{\star *}$ & $.920^{*}$ & & & & & & \\
\hline LLF & $-.483^{* *}$ & $-.287^{* *}$ & $.939^{*}$ & $.923^{*}$ & & & & & \\
\hline FCAT & $.277^{\star \star}$ & $.347^{\star *}$ & $-.270^{* *}$ & $-.237^{*}$ & $-.354^{* *}$ & & & & \\
\hline FCT & .149 & $.290^{\star *}$ & .138 & .166 & .097 & $.298^{\star *}$ & & & \\
\hline FFC & $.372^{\star *}$ & $.245^{\star *}$ & $-.445^{\star}$ & $-.452^{* *}$ & $-.499^{* *}$ & $.215^{\star}$ & -.002 & & \\
\hline FOT & .111 & .153 & -.038 & .043 & -.089 & $.192^{*}$ & $.634^{\star *}$ & .093 & \\
\hline FLRT & $.585^{\star \star}$ & $.531^{* *}$ & $-.623^{* *}$ & $-.555^{\star *}$ & $-.671^{* *}$ & $.523^{\star *}$ & $.420^{\star *}$ & $.558^{\star *}$ & $.423^{\star *}$ \\
\hline $\begin{array}{l}\text { **. La } \\
* \text {. La }\end{array}$ & $\begin{array}{l}\text { elación } \\
\text { elación }\end{array}$ & $\begin{array}{l}\text { signifi } \\
\text { signific }\end{array}$ & $\begin{array}{l}\text { iva en } \\
\text { va en e }\end{array}$ & $\begin{array}{l}\text { hivel } 0,0 \\
\text { ivel } 0,05\end{array}$ & $\begin{array}{l}\text { (bilateral } \\
\text { (bilateral) }\end{array}$ & & & & \\
\hline
\end{tabular}

EOR: estrés en la organización. EAA: estrés auto- administrado. LTF: liderazgo transformacional. LTS: liderazgo transaccional. LLF: Laissez Faire. FCAT: factor condiciones en el ambiente de trabajo. FCT: factor carga de trabajo. FFC: factor fallas de control sobre el trabajo. FOT: factor organización del tiempo de trabajo. FLRT: factor liderazgo y relaciones en el trabajo.

Para que los datos sean significativos se tomó en cuenta la correlación significativa en el nivel 0,01 además de que los resultados deben ser mayores a 0,300 para ser relevantes en los resultados.

En una primera instancia se puede observar en la tabla anterior, la existencia de una correlación significativa del 0,01 entre los tres factores estudiados, lo cual respalda la hipótesis 2 .

En lo referente a la hipótesis 3 se puede decir lo siguiente, tomando en cuenta los resultados de la tabla 2. Es posible observar que tanto en el estrés organizacional (EO) y el estrés auto administrado (EAA), la presencia del LLF muestra una relación más significativa que con los otros dos tipos de liderazgo. Un resultado de gran significancia obtenido dentro de la investigación muestra que cuando los FLRT (factor de liderazgo y relaciones de trabajo) se ven en niveles bajos o nulos (presencia de LLF) los 4 Factores de Riesgo restantes (FCAT, FCT, FFC y FOT). Se presentan en el ambiente de trabajo del personal operativo y administrativo, otro aspecto que presenta un aumento significativo se presentan los dos tipos de estrés EO y EAA.

Por otra parte, se puede observar 
que en cuestiones del LTF y LTS, los resultados significativos son menores de 0,300 , lo cual indica que los niveles tanto de estrés como de FRP se ven disminuidos cuando se ejerce alguno de estos dos tipos de liderazgo, lo cual ayuda a comprobar la hipótesis 4 .

Para llevar a cabo esta intervención se sugiere la utilización del modelo El IMCOC, el cual fue validado en México desde 2015, toma como referencia el marco teórico de las relaciones humanas que se inicia a partir de las investigaciones de Elton Mayo. Este enfoque introduce elementos en la teoría administrativa como son: autoridad, jerarquía, racionalidad, departamentalización, división del trabajo, estándares de producción, supervisión. Estos son complementados con nuevos conceptos como motivación, liderazgo, organización informal, dinámica de grupo, comportamiento organizacional y estilos de liderazgo (Méndez, 2006).

Por su parte para la intervención de los FRP, un reciente documento (Burton, 2010) el cual es redactado por la Organización Mundial de la Salud (OMS), resume las medidas de intervención psicosocial en función de su eficacia, por lo que esta deberá ser elegida por el Centro Universitario en función de las necesidades de sus trabajadores.

Con el fin de tratar el estrés presente en los empleados se sugieren las buenas prácticas sobre estrés laboral (Lamontagne at al., 2007) señalan que las medidas aplicadas son diversas de forma que se utilizan técnicas del diseño del trabajo o el fomento del apoyo social en prevención primaria, o la formación sobre manejo de situaciones conflictivas como medida secundaria. A nivel terciario lo que se pretende es rehabilitar y tratar a los trabajadores que están sufriendo los síntomas relacionados con el estrés. También en este caso se aplica la intervención psicológica para minimizar los efectos del estrés y garantizar la salud laboral y mental del personal del CUSur.

\section{Conclusiones.}

La ejecución del trabajo puede tener efectos positivos 0 negativos para la salud de la persona. Cuando un trabajo es satisfactorio, la persona es motivada, aumenta su capacidad de trabajo, además se mejora la eficacia y la productividad, por otra parte, cuando el trabajo y las exigencias sobrepasan las capacidades del personal se genera ansiedad, negatividad, presencia de estrés laboral y Factores de Riesgo. Cuando no hay una presencia de liderazgo el personal se ve en la necesidad de intervenir por su cuenta en los procesos y al no tener una dirección concreta ni un propósito establecido, el trabajo puede desembocar en estrés o la presencia de factores de riesgo.

La presencia del liderazgo transaccional o transformacional son estilos que traen efectos positivos a las organizaciones, sobre todo hablando del transformacional debido a que es más aplicable en entornos dinámicos que mantienen un proceso de cambio, como lo es una institución educativa. Además, se centra en las necesidades individuales y el crecimiento de sus trabajadores, por lo cual se sugiere la aplicación y desarrollo de este estilo de liderazgo dentro de las instalaciones del CUSur, ya que acorde a los resultados se observó un índice elevado de Laissez Faire.

Dentro de las limitaciones se encuentra el número de participantes, por lo cual en futuras investigaciones 
se sugiere incluir no solo al personal operativo y administrativo, sino también al personal docente de la institución. Además, debido a los resultados se sugiere la aplicación de un programa de capacitación en liderazgo, acorde a las necesidades se la institución y del personal, así como programas de intervención para disminuir los niveles de estrés y los Riesgo Psicosociales.

\section{Referencias bibliográficas:}

Avolio, Bruce; Bass, Bernard (2004), Multifactor Leadership Questionnaire. Third Edition Manual and Sampler Set. Mind Garden, Inc.

Bass, Bernard (1985), Leadership and Performance Beyond Expectations. New York: The Free Press.

Bass, Bernard (1999), Two Decades of Research and Development in Transformational Leadership. European Journal of Work and Organizational Psychology. Vol. VIII, No. 1. Pp. 9-13.

Bateeman, Thomas; Snell, Scott (2001), Administration, una perspectiva global. Cuarta edition. McGraw Hill, México.

Burton, Joan (2010), Healthy Workplace Framework and Model: Background Document and Supporting Literature and Practices. En linea: http://www. who.int/occupational_health/healthy_workplace_framework.pdf

Edison R., Juhro S., Aulia A, et al. (2019). Liderazgo transformacional y Neurofeedback: la perspectiva médica de Neuroleadership. Inter de Liderazgo Organizacional. Vol. 8, No. 1.Pp. 46-62.

Leka Stavroula; Griffiths Amanda; Cox Tom (2004), La organización del trabajo y el estrés. Ginebra: Organización Mundial de la Salud.
Lamonttagne, Anthony; Keegel, Tessa; Louie, Amber; Ostry, Aleck; Landsbergis, Paul (2007), A Systematic Review of the Job-stress Intervention Evaluation Literature. Job-Stress Evaluation. Vol. 13. Pp. 268-280.

Méndez, Carlos (2006), Clima Organizacional en Colombia. El IMCOC: un método de análisis para su intervención. Colombia, Bogotá D.C: Centro Editorial Rosarista.

Mendoza, Ignacio (2005), Estudio diagnóstico del perfil de liderazgo transformacional y transaccional de gerentes de ventas de una empresa farmacéutica a nivel nacional. México: Universidad de Tlaxcala, Tesis doctoral Ciencias Administrativas.

NORMA Oficial Mexicana NOM-035STPS (2018). Factores de riesgo psicosocial en el trabajo-Identificación, análisis y prevención. Estados Unidos Mexicanos. Secretaría del Trabajo y Previsión Social. Martes 23 de octubre 2018, Disponible en: http:// www.dof.gob.mx/nota_detalle.php?codigo $=5541828 \&$ fecha $=23 / 10 / 2018$

Organización Internacional del Trabajo (1998). Declaración Sociolaboral de Mercosur. 1 de Febrero de 1998, disponible en: http://www.ccscs.org/ index.php/documentos-ccscs/participacion-en-el-mercosur/item/534-declaraci \% C 3\% B 3n-socio-laboral-del-mercosur.

Organización Internacional del Trabajo. (2016). El estrés en el trabajo: un reto colectivo;28 DE ABRIL 2016 OIT. Disponible en: http://www.ilo.org/wcmsp5/groups/public/---ed_protect/--protrav/---safework/documents/publication/wcms_466549.pdf.

Organización Mundial de la Salud. (2017). Protección de la salud de los trabajadores. 30 de Noviembre del 2017, Disponible en: https://www. who.int/es/news-room/fact-sheets/ 
detail/protecting-workers'-health.

Romero, Carmen; Beleño, Rosana; Ucros, María; Echeverría, Ana; Lasprilla, Silvana (2016), Factores de riesgos psicosociales extralaborales en personal administrativo universitario. Revista de Enfermería Actual en Costa Rica. Vol. 31, No 4. Pp. 1-15.

Samuel Medina; Lourdes María; Manuel Pando (2007), Adaptación de la escala de estrés laboral organizacional para trabajadores mexicanos. Departamento de salud pública. Centro Universitario de las Ciencias de la Salud. Universidad de Guadalajara. Revista de Salud Pública y Nutrición. Vol. 43, No 4. Pp. 1-18.

Van de Broke Karla; Juliet Hassard; Dani
Flemming; Robert Grundler; Philip Dewe; Kevin Teoh (2015). Calculating the costs of work-related stress and psychosocial risks. En línea: https:// osha.europa.eu/en/tools-and publications/publications/literature_reviews/ calculating-the-cost-of-work-related-stress-and-psychosocial-risks

Viera, Verónica (2014), El estrés laboral y rendimiento de los trabajadores de la empresa Megaprofer S.A Ambato. Repositorio Universidad Técnica de Ambato. Vol. 43, No 4. Pp. 1-18. En línea: http://repo.uta.edu.ec/ handle/123456789/7790

Villegas, J. (1996). Desarrollo Gerencial. Enfoque Conceptual y Metodología. Editorial Texto, Caracas Venezuela.

- Esta obra está bajo una licencia de Creative Commons Reconocimiento-NoComercialCompartirlgual 3.0 Unported.

http://creativecommons.org/licenses/by-nc-sa/3.0/deed.es_ES 